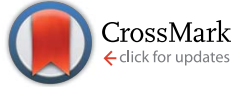

Cite this: RSC Adv., 2016, 6, 56091
Received 25th March 2016 Accepted 3rd June 2016

DOI: 10.1039/c6ra07771d

www.rsc.org/advances

\section{Co-cultivation of Synechocystis salina and Pseudokirchneriella subcapitata under varying phosphorus concentrations evidences an allelopathic competition scenario $\uparrow$}

\author{
A. L. Gonçalves, ${ }^{a}$ A. C. Abreu, ${ }^{a}$ A. Coqueiro, ${ }^{b}$ A. Gaspar, ${ }^{c}$ F. Borges, ${ }^{c}$ Y. H. Choi, ${ }^{b}$ \\ J. C. M. Pires ${ }^{a}$ and M. Simões ${ }^{\star a}$
}

Microalgae and cyanobacteria have received ample attention in the last few decades due to their environmental and biotechnological applications. Co-cultures of these microorganisms may present benefits particularly on wastewater bioremediation and biomass production. However, the understanding on the interactions between photosynthetic microorganisms is still in an early stage of knowledge. In this line, the aim of the present study was the evaluation of the growth dynamics of co-cultures of a cyanobacterium, Synechocystis salina, and a microalga, Pseudokirchneriella subcapitata, under low phosphate-phosphorus concentrations. Kinetic growth parameters were determined through the Monod and modified Gompertz models and evidence of allelochemical production was confirmed through metabolomic analysis of the supernatant obtained from the co-cultures using GC-MS and 1D-NMR. Kinetic growth parameters have shown that $P$. subcapitata was better adapted to grow under low phosphorus concentrations. Co-cultivation of these microorganisms did not influence $P$. subcapitata growth; however, S. salina growth was strongly inhibited. The modified Gompertz model has shown that growth inhibition of $S$. salina in co-cultures may be related to the activity of allelochemicals produced by P. subcapitata. This assumption was corroborated by the assessment of the antimicrobial potential of lactic acid (2-hydroxypropanoic acid), an organic acid identified in the supernatant from the co-cultures with growth inhibitory effects against $S$. salina.

\section{Introduction}

Microalgal/cyanobacterial culturing has been the focus of several research studies worldwide due to the huge biotechnological potential of these photosynthetic microorganisms., ${ }^{\mathbf{1}, 2}$ When growing autotrophically, microalgae and cyanobacteria perform photosynthesis converting $\mathrm{CO}_{2}$ (from the atmosphere or flue gas emissions) into organic carbon compounds, thus reducing $\mathrm{CO}_{2}$ accumulation in the atmosphere. ${ }^{3-6}$ Additionally, these microorganisms can assimilate nutrients, such as nitrogen and phosphorus species released into the environment and frequently found in wastewaters, meaning that they can be applied in wastewater treatment processes. ${ }^{7-10}$ Furthermore,

${ }^{a}$ LEPABE, Departamento de Engenharia Quimica, Faculdade de Engenharia, Universidade do Porto, Rua Dr. Roberto Frias, 4200-465 Porto, Portugal. E-mail: mvs@fe.up.pt; Fax: +351 22508 1449; Tel: +351 225081654

${ }^{b}$ Natural Products Laboratory, Institute of Biology, Leiden University, Leiden, The Netherlands

${ }^{c}$ CIQUP, Department of Chemistry and Biochemistry, Faculty of Sciences, University of Porto, Rua do Campo Alegre s/n, 4169-007, Porto, Portugal

$\dagger$ Electronic supplementary information (ESI) available. See DOI: 10.1039/c6ra07771d microalgal/cyanobacterial biomass has other diverse attractive applications, ${ }^{\mathbf{1} 11-13}$ particularly human food and animal feed, production of cosmetics, drugs, functional food and biofuels.

Although the majority of research studies using microalgae and cyanobacteria refer to mono-cultures, several studies have reported the use of microalgal/cyanobacterial co-cultures for diverse applications ${ }^{\mathbf{1 4 - 1 6}}$ namely: (i) biomass production and $\mathrm{CO}_{2}$ uptake in adverse conditions; (ii) pollutant removal from wastewaters; (iii) carbohydrate accumulation for biofuels production; (iv) production of high-valued secondary metabolites; and (v) bio-flocculation and biofilm formation. The use of co-cultures combining microorganisms presenting different metabolic activities and adapted to different environmental conditions results in the development of a robust system that can operate under different environmental conditions and different nutrient supplies. ${ }^{\mathbf{1 7 - 1 9}}$ Therefore, important characteristics of these cultures include: (i) high tolerance to environmental fluctuations and to multiple nutrient sources; and (ii) resistance to invasion by other species. However, due to the huge number of possible combinations between these microorganisms, studies on multispecies growth are still in an early stage of knowledge. 
Furthermore, the study of interactions between different microalgal species or between microalgae and cyanobacteria is of great importance to understand their behaviour in aquatic environments. Aquatic photoautotrophs often face severe competition for resources, either space, light or nutrients. ${ }^{15,20}$ In these competitive environments, microorganisms tend to produce secondary metabolites, known as allelochemicals. The biosynthesis pathways and mode of action of these compounds, also identified as the chemical ecology of microalgae, has received much attention in the last few years, due to their importance in natural products chemistry and in several biotechnological processes, such as bioremediation and wastewater treatment. ${ }^{\mathbf{1 4 , 2 1}}$

Allelopathy is defined as the direct or indirect harmful effect of one species on another through the production of chemicals released to the environment. It occurs essentially under stress situations, such as nutrient limitation. Target organisms might be more susceptible to allelochemicals under stress, and/or donor organisms might induce or increase the production of allelopathically active compounds in such conditions. ${ }^{2021}$ For example, polyphenolic compounds produced by some organisms interfere with alkaline phosphatase, an exoenzyme used by several algae and cyanobacteria to overcome phosphorus limitation. ${ }^{20}$

To better understand the behaviour of photosynthetic organisms in aquatic environments, mathematical models have been developed to describe microalgal/cyanobacterial growth. ${ }^{22,23}$ The majority of these models are mainly applied to mono-cultures and in laboratory environments. ${ }^{24}$ Therefore, these type of models need to be adapted to allow their application to more complex systems, such as co-cultures of photosynthetic microorganisms.

This study provides an experimental and mathematical approach towards the understanding of the interactions between Synechocystis salina and Pseudokirchneriella subcapitata when exposed to a stress condition (low phosphate-phosphorus concentrations), trying to overcome the limitations of current mathematical models that can only be applied to microalgal/ cyanobacterial mono-cultures. The specific aims of this study were: (i) to characterize the growth dynamics of mono- and cocultures of these microorganisms when grown under limiting phosphorus concentrations; (ii) to establish a mathematical model able to describe the behaviour of these microorganisms in mono- and co-cultures; and (iii) to evaluate possible allelopathic interactions between these microorganisms. Phosphorus is one of the most important macronutrients for microalgae and cyanobacteria, as this nutrient is used for the synthesis of proteins, nucleic acids and phospholipids. ${ }^{25,26}$ Accordingly, microalgal/cyanobacterial cultures were supplied with low concentrations of this nutrient to evaluate possible growth competition between the studied microorganisms. Selection of the microorganisms integrating the co-cultures is a critical step. One possible alternative is to combine, for example, photoautotrophs and mixotrophs, ammonia and nitrate users, or marine and freshwater, aiming to improve both biomass productivities and the resilience of the co-culture. ${ }^{18}$ In this study, a marine cyanobacterium, $S$. salina, was co-cultured with a freshwater microalga, P. subcapitata. Selection of a marine microorganism was based on the following factors: ${ }^{18}$ (i) marine microalgae or cyanobacteria are more resilient to salinity changes and can be cultured in freshwater; and (ii) the high productivities observed in marine coastal waters, even when submitted to considerable salinity and nutrient oscillations, suggest that these microorganisms may be effectively used for biomass production using wastewaters as culture medium. $P$. subcapitata is a green microalga that has shown to easily adapt to grow under low phosphorus concentrations. ${ }^{21}$ Additionally, several authors have reported the use of both $S$. salina and $P$. subcapitata a wide variety of biotechnological applications, such as wastewater treatment ${ }^{27}$ and synthesis of bioactive compounds. $^{18}$

\section{Materials and methods}

\subsection{Microorganisms and culturing conditions}

S. salina LEGE 06079 was obtained from the Laboratory of Ecotoxicology, Genomic and Evolution (LEGE) - CIIMAR (Centre of Marine and Environmental Research of the University of Porto, Porto, Portugal) and P. subcapitata 278/4 was obtained from the Culture Collection of Algae and Protozoa (CCAP, Scotland, UK). Stock solutions of these microorganisms were prepared in OECD test medium (Organisation for Economic Co-operation and Development ${ }^{28}$ ), a synthetic medium commonly used for microalgal/ cyanobacterial growth. ${ }^{29-31}$ Culture medium was sterilized by autoclaving at $121{ }^{\circ} \mathrm{C}$ for $15 \mathrm{~min}$. Cultures were incubated in $500 \mathrm{~mL}$ flasks at room temperature $\left(25 \pm 2{ }^{\circ} \mathrm{C}\right)$, under continuous exposure to fluorescent light with irradiance of approximately $72 \mu \mathrm{E} \mathrm{m} \mathrm{m}^{-2} \mathrm{~s}^{-1}$. Atmospheric air (filtered through $0.22 \mu \mathrm{m}$ cellulose acetate membranes, Orange Scientific, Braine-l'Alleud, Belgium) was bubbled at the bottom of the flasks to promote agitation.

\subsection{Mono- and co-cultures growth under different phosphorus concentrations}

Batch experiments with mono- and co-cultures were performed to study the influence of low phosphate-phosphorus $\left(\mathrm{KH}_{2} \mathrm{PO}_{4}\right.$, Sigma-Aldrich, St. Louis, MO, USA) concentrations (1.50 to 24.0 $\times 10^{-3} \mathrm{mg} \mathrm{L}^{-1}$ of $\mathrm{KH}_{2} \mathrm{PO}_{4}$, which corresponds to 0.341 to $5.46 \times$ $10^{-3} \mathrm{mg}_{\mathrm{P}} \mathrm{L}^{-1}$ of phosphate-phosphorus) on $S$. salina and $P$. subcapitata growth dynamics. Selection of this concentration range was based on the one reported by Fergola et al. ${ }^{21}$ when evaluating allelopathic competition between Chlorella vulgaris and $P$. subcapitata. After an acclimation period of seven days under these concentrations, microorganisms were cultured for twelve days in $500 \mathrm{~mL}$ flasks (working volume of $400 \mathrm{~mL}$ ), with an initial cell concentration of about 1.0 to $2.0 \times 10^{6}$ cells per $\mathrm{mL}$. Other growth conditions, such as light, temperature and aeration, were similar to those previously described. Two independent experiments were performed for each studied condition. 


\subsection{Determination of $S$. salina and $P$. subcapitata growth parameters}

Specific growth rates $\left(\mu, \mathrm{h}^{-1}\right)$ were determined by the evaluation of cell concentration within the cultivation time. These assays were performed in duplicate using a Neubauer counting chamber (Marienfeld, Lauda-Königshofen, Germany) and a Leica DM LB (Leica Microsystems, Wetzlar, Germany) microscope. The relationship between cell and biomass concentrations was obtained by determination of cell dry weight of both microorganisms for different cell concentrations and established through linear regression $\left(R^{2} \geq 0.995\right.$; data not shown). Specific growth rates were determined according to eqn (1): ${ }^{32}$

$$
\mu=\frac{\ln X_{2}-\ln X_{1}}{t_{2}-t_{1}}
$$

where $X_{2}$ and $X_{1}$ correspond to biomass concentration (in $\mathrm{mg}$ $\mathrm{L}^{-1}$ ) at times $t_{2}$ and $t_{1}$ (the end and beginning of exponential growth phase, in $\mathrm{h}$, respectively).

Average biomass productivities $\left(P, \mathrm{mg} \mathrm{L}^{-1} \mathrm{~h}^{-1}\right)$ were calculated from the variation in biomass concentration within the cultivation time, as shown in eqn (2): ${ }^{32,33}$

$$
P=\frac{X_{\mathrm{f}}-X_{\mathrm{i}}}{t_{\mathrm{f}}-t_{\mathrm{i}}}
$$

where $X_{\mathrm{f}}$ and $X_{\mathrm{i}}$ correspond to biomass concentration (in $\mathrm{mg}$ $\mathrm{L}^{-1}$ ) at times $t_{\mathrm{f}}$ and $t_{\mathrm{i}}$ (the end and beginning of cultivation time, in $\mathrm{h}$, respectively).

\subsection{Kinetic modelling of specific growth rates from mono- and co-cultures}

Specific growth rates determined for each phosphatephosphorus concentration assessed $\left(S, \mathrm{mg}_{\mathrm{P}} \mathrm{L}^{-1}\right)$ were used to determine the kinetic parameters $\mu_{\max }$ (maximum specific growth rate, $\mathrm{h}^{-1}$ ) and $K_{\mathrm{S}}$ (half saturation constant, $\mathrm{mg}_{\mathrm{P}} \mathrm{L}^{-1}$ ), according to the Monod model: ${ }^{34}$

$$
f(S)=\frac{\mu_{\max } S}{K_{\mathrm{S}}+S}
$$

The use of the Monod model to predict microalgal and cyanobacterial growth in response to varying phosphorus concentrations was selected based on previous reports describing the effective use of this model to evaluate phytoplankton growth kinetics. $^{35-37}$

\subsection{Kinetic modelling of allelopathic-based competition in co-cultures}

As the kinetic growth parameters determined through the Monod model have shown that the growth of $S$. salina in cocultures may be limited by other factors rather than nutrient limitation, the growth of both microorganisms in mono- and co-cultures was evaluated using a modified version of the Gompertz model: ${ }^{38}$

$$
y=a \exp [-\exp (b-c t)]
$$

where $y$ is the output value, $a$ is the upper asymptote, $b(b>0)$ sets the displacement along the $x$ axis and $c(c>0)$ sets the tangent at the inflection point. The Gompertz model was selected in this study because several authors have already reported the use of this model to predict microalgal and cyanobacterial growth, evidencing that it sufficiently predicted the growth of Scenedesmus obliquus, ${ }^{39}$ Spirulina platensis ${ }^{22}$ and Aphanothece microscopica Nägeli. ${ }^{40}$ By substituting the parameters $a, b$ and $c$ (see ESI, File $\mathrm{S} 1 \dagger$ ), the modified Gompertz model was obtained:

$$
X=A \exp \left[-\exp \left(\mu_{\max }(\lambda-t)+1\right)\right]
$$

where $\lambda$ is the lag time (in h) and $A$ is the highest biomass concentration (in $\mathrm{mg} \mathrm{L}^{-1}$ ) achieved. Specific growth rates were considered as a function of phosphate-phosphorus concentration in the culture medium. For that, the Monod model already determined for both microorganisms was used. To assess the temporal variation of phosphorus and biomass concentrations of mono-cultures two differential equations (eqn (6)) were defined as following:

$$
\left\{\begin{array}{c}
\frac{\mathrm{d} S}{\mathrm{~d} t}=-\alpha \frac{\mathrm{d} X}{\mathrm{~d} t} \\
\frac{\mathrm{d} X}{\mathrm{~d} t}=A f(S) \exp [-\exp (f(S)(\lambda-t)+1)] \exp (f(S)(\lambda-t)+1)
\end{array}\right.
$$

where $\alpha$ corresponds to the mass fraction of phosphorus in the biomass. In the calculations, it was assumed that the mass fraction of phosphorus in the biomass was $0.01 \%$, considering the typical molecular formula of microalgal biomass: $\mathrm{CO}_{0.48} \mathrm{H}_{1.83} \mathrm{~N}_{0.11} \mathrm{P}_{0.01}{ }^{41}$ The differential equations were integrated using the fourth-order Runge-Kutta method, as described by Chapra and Canale. ${ }^{42}$

As experimental data has shown that the growth of $S$. salina in co-cultures was strongly influenced by the presence of $P$. subcapitata, the model was adapted by including the parameters $\gamma$ and $\beta$ proposed by Fergola et al. ${ }^{\mathbf{2 1}}$ Therefore, it was assumed that the microalga produced allelochemicals towards the cyanobacterium and that the specific growth rate of the cyanobacterium decreased for increasing concentrations of allelochemicals, undergoing a function of type:

$$
\mu_{1}(S)=f_{1}(S) \mathrm{e}^{-\gamma X_{2}}
$$

where $\mu_{1}(S)$ is the specific growth rate $\left(\right.$ in $^{-1}{ }^{-1}$ ) of $S$. salina in cocultures, $f_{1}(S)$ corresponds to the function determined by the Monod model (eqn (3)) for S. salina grown in mono-cultures, $\gamma(>0)$ denotes a measure of the inhibitory effect of the allelochemicals produced by $P$. subcapitata and $X_{2}$ corresponds to the concentration of $P$. subcapitata (in $\mathrm{mg} \mathrm{L}^{-1}$ ) at time $t$. On the other hand, $P$. subcapitata growth in co-cultures was defined as:

$$
\mu_{2}(S)=f_{2}(S)(1-\beta)
$$

where $\mu_{2}(S)$ is the specific growth rate (in $\mathrm{h}^{-1}$ ) of $P$. subcapitata in co-cultures, $f_{2}(S)$ corresponds to the function determined by the Monod model (eqn (3)) for P. subcapitata grown in mono- 
cultures and $\beta(0<\beta<1)$ denotes the fraction of potential growth devoted to allelochemicals production.

The modified Gompertz model established in eqn (5), as well as the assumptions expressed in eqn (7) and (8), resulted in a three-equation system, which was used to model the phosphorus uptake and the growth of both $S$. salina and $P$. subcapitata in co-cultures:
The oven temperature was increased to $50{ }^{\circ} \mathrm{C}$ and held at this temperature for $2 \mathrm{~min}$. Then, temperature was raised to $250{ }^{\circ} \mathrm{C}$ at a rate of $8{ }^{\circ} \mathrm{C} \min ^{-1}$, to $300{ }^{\circ} \mathrm{C}$ at a rate of $3{ }^{\circ} \mathrm{C} \mathrm{min}^{-1}$ and to $310{ }^{\circ} \mathrm{C}$ at a rate of $3{ }^{\circ} \mathrm{C} \mathrm{min}^{-1}$. Total run time was $47 \mathrm{~min}$.

Data processing. Registered peaks were identified by comparison with the mass spectra available in the National Institute of Standards and Technology (NIST) library.

$$
\left\{\begin{array}{c}
\frac{\mathrm{d} S}{\mathrm{~d} t}=-\alpha_{1} \frac{\mathrm{d} X_{1}}{\mathrm{~d} t}-\alpha_{2} A_{2} f_{2}(S) \exp \left[-\exp \left(f_{2}(S)\left(\lambda_{2}-t\right)+1\right)\right] \exp \left(f_{2}(S)\left(\lambda_{2}-t\right)+1\right) \\
\frac{\mathrm{d} X_{1}}{\mathrm{~d} t}=A_{1} \mu_{1}(S) \exp \left[-\exp \left(\mu_{1}(S)\left(\lambda_{1}-t\right)+1\right)\right] \exp \left(\mu_{1}(S)\left(\lambda_{1}-t\right)+1\right) \\
\frac{\mathrm{d} X_{2}}{\mathrm{~d} t}=A_{2} \mu_{2}(S) \exp \left[-\exp \left(\mu_{2}(S)\left(\lambda_{2}-t\right)+1\right)\right] \exp \left(\mu_{2}(S)\left(\lambda_{2}-t\right)+1\right)
\end{array}\right.
$$

where $\alpha_{1}$ and $\alpha_{2}$ correspond to the mass fraction of phosphorus in $S$. salina and $P$. subcapitata cells, respectively.

The parameters $\lambda_{1}, \lambda_{2}, A_{1}$ and $A_{2}$, previously determined for mono-cultures, were applied in this system to allow the determination of $\gamma$ and $\beta$. Integration of these equations was also performed using the fourth-order Runge-Kutta method. ${ }^{\mathbf{4 2}}$

The model fits of the Monod and modified Gompertz models were obtained through nonlinear regression techniques and the estimated parameters were determined using an iterative procedure that minimizes the sum of squared residuals. The quality of the model fits was evaluated by calculating the performance indexes described by Queiroz et al.: ${ }^{43}$ (i) root mean squared error (RMSE); (ii) standard error of prediction (\% SEP); (iii) bias factor $\left(B_{\mathrm{f}}\right)$; and (iv) accuracy factor $\left(A_{\mathrm{f}}\right)$ (see ESI, File $\mathrm{S} 2 \dagger)$.

\subsection{Analytical methods for allelochemicals identification}

2.6.1. Sample preparation. After the cultivation time, duplicate samples were collected from the flasks corresponding to $S$. salina and $P$. subcapitata co-cultures. These samples were centrifuged at $2900 \mathrm{~g}$ for $15 \mathrm{~min}$ in an Eppendorf $5810 \mathrm{R}$ centrifuge (Eppendorf, Hamburg, Germany) and the supernatant was lyophilized in a Snijders Scientific freeze-dryer (Snijders, Tilburg, Netherlands). The supernatant was then analysed by gas chromatography-mass spectrometry (GC-MS) and onedimensional nuclear magnetic resonance (1D-NMR), as described by $\mathrm{Li}$ and $\mathrm{Hu}^{44}$ and $\mathrm{Ni}$ et $a .^{45}$

\subsubsection{GC-MS analysis}

Instrumentation. GC-MS analysis was performed on an Agilent Technologies 7890A gas chromatograph coupled to a 5975C mass selective detector (Agilent Technologies, Palo Alto, CA, USA). The mass spectra were obtained by electron ionization at $70 \mathrm{eV}$.

Chromatographic conditions. DB-5 capillary column (crosslinked, 5\% diphenyl, 95\% dimethyl polysiloxane, $30 \mathrm{~m} \times 0.25$ $\mathrm{mm} \times 0.25 \mu \mathrm{m}$, Agilent Technologies Inc., Santa Clara, CA, USA). Helium was used as the carrier gas at a flow rate of $1 \mathrm{~mL}$ $\min ^{-1}$. The injection volume was $1 \mu \mathrm{L}$ and split ratio was $20: 1$.
Derivatization conditions. An aliquot of the sample (2.5 mg) was transferred into a vial and $75 \mu \mathrm{L}$ of pyridine followed by $75 \mu \mathrm{L}$ of $N, O$-bis(trimethylsilyl)trifluoroacetamide (Alfa Aesar, Ward Hill, MA, USA) containing 1\% trimethyl chlorosilane was added. The derivatization was allowed to occur, firstly, at $60{ }^{\circ} \mathrm{C}$ for $1 \mathrm{~h}$ and then at $40{ }^{\circ} \mathrm{C}$ for $30 \mathrm{~min}$.

\subsubsection{NMR analysis}

Instrumentation. NMR spectra were recorded at room temperature on a $600 \mathrm{MHz}$ DMX-600 spectrometer (Brucker, Karlsruhe, Germany) operating at a proton NMR frequency of 600.13 MHz. Methanol- $d_{4}$ was used as the internal lock. The resulting spectra were manually phased, baseline corrected and calibrated to the internal standard, trimethylsilylpropionic acid sodium salt at $\delta 0.0$ using TOPSPIN software (version 2.0, Bruker).

Sample preparation. The lyophilized material was placed in a $1.5 \mathrm{~mL}$ microtube and dissolved in $1 \mathrm{~mL}$ of a mixture $(1: 1)$ containing methanol- $d_{4}$ and $\mathrm{KH}_{2} \mathrm{PO}_{4}$ buffer ( $\mathrm{pH}$ 6.0) dissolved in $\mathrm{D}_{2} \mathrm{O}$ containing $0.29 \mathrm{mM}$ 3-(trimethylsilyl)propionic acid sodium salt (Sigma Aldrich, St. Louis, MO, USA). The mixture was vortexed at room temperature for $1 \mathrm{~min}$, ultrasonicated for $15 \mathrm{~min}$ in a Branson 5510E-MT ultrasonic cleaner (Branson Ultrasonics, Danbury, CT, USA) and centrifuged at $17000 \mathrm{~g}$ for $20 \mathrm{~min}$ in a Thermo Scientific Heraeus Pico 17 centrifuge (Fischer Scientific, Landsmeer, Netherlands). An aliquot $(0.3 \mathrm{~mL})$ of the supernatant was transferred to a $3 \mathrm{~mm}$ NMR glass tube and analysed.

Data processing. The signals detected in the spectra were analysed by spectral patterns and intensities. After statistical analysis, compounds were identified by comparison of spectral patterns of enrichment and depletion found in the following metabolomic database libraries: Chenomx NMR Suite (Chenomx Inc.) and Leiden University - Natural Products Laboratory (private).

\subsection{Evaluation of the inhibitory activity of identified allelochemicals}

After analysing co-cultures medium, some allelochemicals, particularly organic acids, were selected (2-hydroxypropanoic 
acid (5), butanedioic acid (16), 4-aminobutanoic acid (21) and 2,3,4-trihydroxybutanoic acid (22)) to assess their growth inhibitory potential against $S$. salina and $P$. subcapitata. Stock solutions of the selected organic acids, obtained from Sigma Aldrich (St. Louis, MO, USA), were prepared in sterilized distilled water at a concentration of $1000 \mu \mathrm{g} \mathrm{mL}^{-1}$.

The growth inhibition caused by the selected organic acids was evaluated according to the Bauer et al. ${ }^{46}$ disc diffusion method. Suspensions of $S$. salina and P. subcapitata in the exponential growth phase were harvested, washed twice and resuspended in saline solution $(0.85 \% \mathrm{w} / \mathrm{v} \mathrm{NaCl})$ to obtain a final concentration of about $5.0 \times 10^{6}$ cells per $\mathrm{mL}$. The suspensions were seeded in Petri dishes (90 $\mathrm{mm}$ diameter) containing modified Bold's Basal Medium ${ }^{24}$ supplemented with agar. Sterile filter paper discs (6 $\mathrm{mm}$ diameter) impregnated with approximately $1 \mathrm{mg}$ of the organic acid solutions $(1000 \mu \mathrm{g}$ $\mathrm{mL}^{-1}$ ) were placed in Petri dishes. Afterwards, these Petri dishes were incubated for one week at room temperature under continuous light supply $\left(72 \mu \mathrm{E} \mathrm{m}^{-2} \mathrm{~s}^{-1}\right)$. The clear zones around the discs were recorded. Three independent experiments were performed.

\subsection{Statistical analysis}

Results were expressed as the mean value \pm standard error of the mean. Statistical analysis of experimental data were carried out at a significance level of 0.05 using paired-samples $t$-test from the statistical software SPSS 22.0 (SPSS Inc., Chicago, IL, USA).

\section{Results and discussion}

\subsection{Influence of phosphorus concentrations on $S$. salina and $P$. subcapitata growth parameters}

Specific growth rates and average biomass productivities determined for mono- and co-cultures of S. salina and P. subcapitata grown under different phosphate-phosphorus concentrations are presented in Table 1 (the respective growth curves are presented in ESI, File S3 $\dagger$ ). In general, higher specific growth rates were observed for increasing phosphorus concentrations $(p<0.05)$. These results are in agreement with those reported by Litchman et al. ${ }^{47}$ for the microalgae Nitzschia sp. and Sphaerocystis schroeteri and the cyanobacterium Phormidium luridum. Specific growth rates of $P$. subcapitata were significantly higher $(p<0.05)$ than those of $S$. salina in both mono- and co-cultures. In mono-cultures, specific growth rates for the microalga ranged from $(0.821 \pm 0.115) \times 10^{-2}$ to $(2.87 \pm 0.13) \times 10^{-2} \mathrm{~h}^{-1}$, while for the cyanobacterium ranged from $(0.296 \pm 0.071) \times$ $10^{-2}$ to $(1.59 \pm 0.20) \times 10^{-2} \mathrm{~h}^{-1}$. Lower specific growth rates determined for $S$. salina suggest that low phosphorus concentrations favour the growth of $P$. subcapitata. Similar orders of magnitude were described for two different strains of the cyanobacterium Trichodesmium sp. grown under phosphatephosphorus concentrations ranging from 0 to $20 \mu \mathrm{M} \cdot{ }^{48}$ No significant differences $(p>0.05)$ were found on the specific growth rates determined for $P$. subcapitata grown in mono- and co-cultures. On the other hand, specific growth rates of $S$. salina in co-cultures were statistically lower $(p<0.05)$ than those determined in mono-cultures. These results indicate that cocultivation with $P$. subcapitata is prejudicial to cyanobacterial growth. For diverse phosphorus concentrations higher average biomass productivities were determined for the highest nutrient concentrations. Additionally, average biomass productivities determined for $P$. subcapitata (ranging between $(0.641 \pm 0.134) \times 10^{-2}$ and $\left.(2.54 \pm 0.08) \times 10^{-2} \mathrm{mg} \mathrm{L}^{-1} \mathrm{~h}^{-1}\right)$ were statistically higher $(p<0.05)$ than those determined for $S$. salina (ranging between $(0.119 \pm 0.032) \times 10^{-2}$ and $(0.413 \pm$ $\left.0.028) \times 10^{-2} \mathrm{mg} \mathrm{L}^{-1} \mathrm{~h}^{-1}\right)$. Comparing mono- and co-cultures, average biomass productivities determined for both $S$. salina and $P$. subcapitata grown in mono-cultures were higher than those determined in co-cultures. These results indicate that in co-cultures, lower phosphorus availability leads to lower average biomass productivities, proposing the inadequacy of these co-cultures when large biomass amounts are required. Average biomass productivities determined in mono- and cocultures of $S$. salina presented a similar behaviour to the one observed for specific growth rates. In P. subcapitata cultures, average biomass productivities contrast with specific growth rate values, which have shown to be similar $(p>0.05)$ in both

Table 1 Specific growth rates $\left(\mu\right.$, in $\left.\mathrm{h}^{-1}\right)$ and average biomass productivities $\left(P\right.$, in $\left.\mathrm{mg} \mathrm{L}^{-1} \mathrm{~h}^{-1}\right)$ determined for mono- and co-cultures of $S$. salina and $P$. subcapitata grown under different phosphorus concentrations $\left(S \text {, in } \mathrm{mg}_{\mathrm{P}} \mathrm{L}^{-1}\right)^{a}$

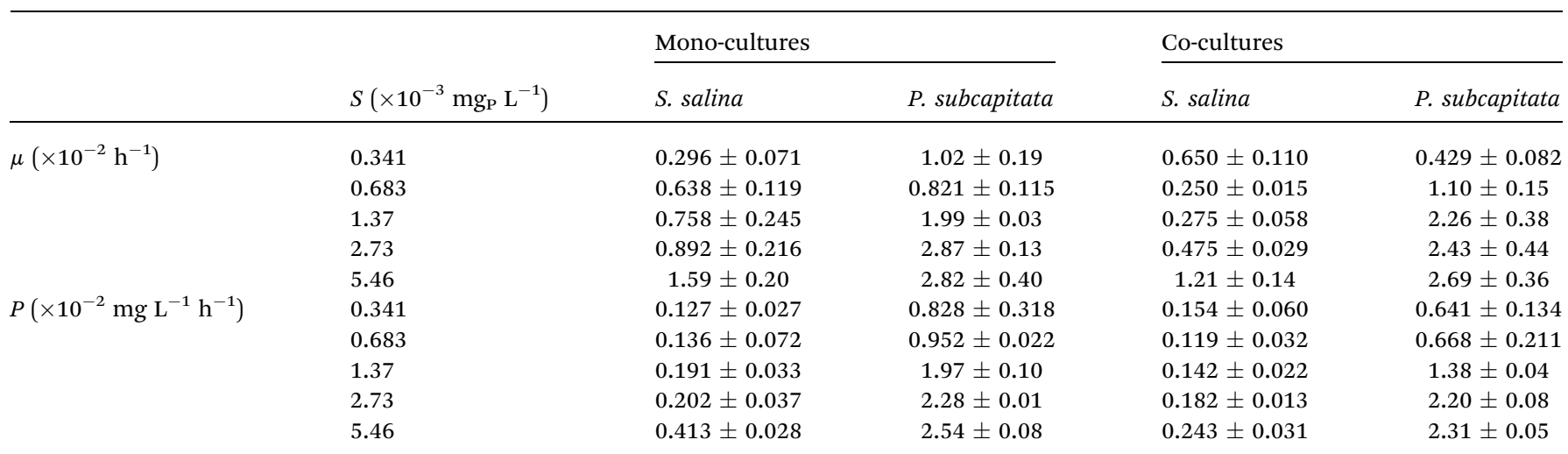

${ }^{a}$ Values are presented as the mean \pm standard error of the mean of two independent experiments. 
mono- and co-cultures. Inhibitory growth effects in co-cultures of microalgae has already been reported in the literature. For example, Solé et $a .^{40}$ have reported growth inhibition of Heterocapsa triquetra when co-cultured with Chrysocromulina polylepis. The mechanisms involved in the inhibitory effects of $C$. polylepis remain unknown.

\subsection{Kinetic modelling of specific growth rates from mono- and co-cultures}

Specific growth rates obtained for the different phosphatephosphorus concentrations were used to establish a model fit (Fig. 1) according to the hyperbolic Monod function (eqn (3)) and to determine the associated kinetic growth parameters (Table 2). The quality of the model fits was evaluated through the performance indexes presented in Table 2 . The low values determined for RMSE and \% SEP as well as $B_{\mathrm{f}}$ and $A_{\mathrm{f}}$ values of approximately 1 have shown that the models are able to accurately describe the relationship between specific growth rates and phosphorus concentrations in the culture medium. As phosphorus concentration increases, there is an increase in

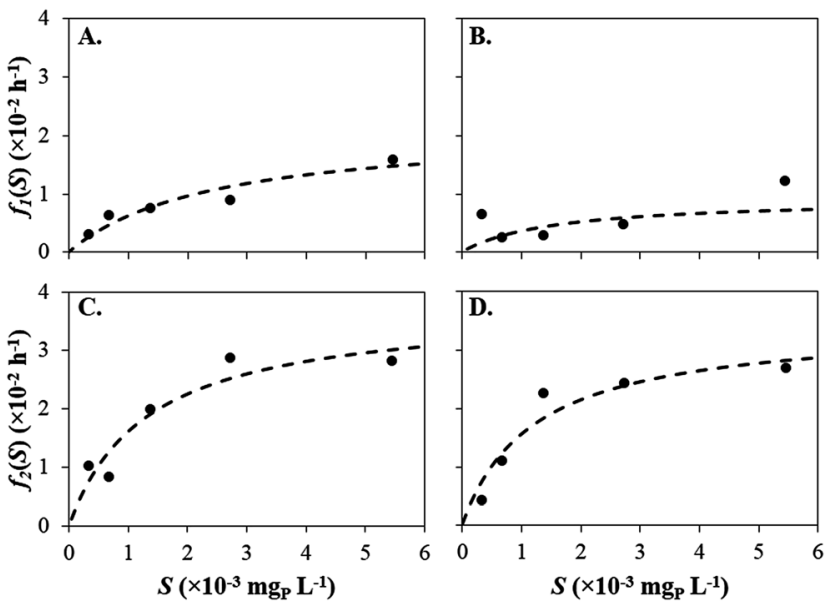

Fig. 1 Model fit of the Monod model to the experimental data: (A) S. salina grown in mono-cultures; (B) S. salina grown in co-cultures; (C) P. subcapitata grown in mono-cultures; (D) P. subcapitata grown in co-cultures. Dashed lines represent the predicted values obtained through the Monod model. specific growth rates until a certain concentration, where this kinetic parameter remains approximately constant (Fig. 1). Similar results were obtained for P. subcapitata and Trichodesmium sp. in the studies performed by Fergola et al. ${ }^{21}$ and $\mathrm{Fu}$ et al. ${ }^{48}$ respectively. The maximum specific growth rates determined for $P$. subcapitata in mono- and co-cultures were not statistically different $(p>0.05)$. However, they were significantly lower $(p<0.05)$ for $S$. salina, suggesting that low phosphorus concentrations can be a growth limiting factor to this microorganism. Additionally, $\mu_{\max }$ determined for $S$. salina grown in co-cultures was statistically lower $(p<0.05)$, meaning that these conditions favoured the growth of $P$. subcapitata. Lower $K_{\mathrm{S}}$ values obtained for the microalga indicate that this organism is better adapted to uptake phosphate-phosphorus supplied at low concentrations. On the other hand, higher $K_{\mathrm{S}}$ values estimated for $S$. salina indicate that the growth of this strain may be limited by phosphorus concentration. However, half saturation constant determined for the cyanobacterium in co-cultures $\left(1.57 \pm 0.26 \times 10^{-3} \mathrm{mg}_{\mathrm{P}} \mathrm{L}^{-1}\right)$ was statistically lower $(p<0.05)$ than the one obtained for mono-cultures $\left(2.45 \pm 0.40 \times 10^{-3}\right.$ $\mathrm{mg}_{\mathrm{P}} \mathrm{L}^{-1}$ ), indicating that the growth of $S$. salina in co-cultures may be limited by other factors rather than phosphorus limitation.

\subsection{Kinetic modelling of allelopathic-based competition in co-cultures}

As the kinetic parameters determined through the model fit of the Monod function suggested that the growth of the cyanobacterium in co-cultures may be inhibited by other factors rather than phosphorus limitation, a new model was established to describe the behaviour of both microorganisms (in mono- and co-cultures). The new model, which was based on the Gompertz model, takes into account the hypothesis that $S$. salina growth inhibition can be related to the presence of allelochemicals excreted by $P$. subcapitata. The use of the Gompertz model to describe microalgal and bacterial growth has already been reported in the literature. ${ }^{22,39,40,49}$ In this study, the referred model was adapted by assuming that $S$. salina growth decreased in response to increased concentrations of the allelochemicals produced by $P$. subcapitata and that $P$. subcapitata presented a fraction of potential growth devoted

Table 2 Kinetic parameters and performance indexes of the Monod model for mono- and co-cultures of S. salina and $P$. subcapitata ${ }^{a}$

\begin{tabular}{lllll}
\hline & Mono-cultures & & Co-cultures \\
\cline { 2 - 3 } & S. salina & P. subcapitata & & S. salina \\
\hline$\mu_{\max }\left(\times 10^{-2} \mathrm{~h}^{-1}\right)$ & $2.13 \pm 0.56$ & $3.75 \pm 0.71$ & $0.932 \pm 0.198$ & P. subcapitata \\
$K_{\mathrm{S}}\left(\times 10^{-3} \mathrm{mg}_{\mathrm{P}} \mathrm{L}^{-1}\right)$ & $2.45 \pm 0.40$ & $1.32 \pm 0.67$ & $1.57 \pm 0.26$ & $3.47 \pm 0.59$ \\
$\operatorname{RMSE}\left(\times 10^{-2} \mathrm{~h}^{-1}\right)$ & 0.14 & 0.29 & 0.321 & $1.22 \pm 0.57$ \\
$\% \mathrm{SEP}$ & 17 & 16 & 56 & 14 \\
$B_{\mathrm{f}}$ & 0.943 & 1.01 & 0.802 & 1.11 \\
$A_{\mathrm{f}}$ & 1.17 & 1.21 & 1.71 & 1.21
\end{tabular}

${ }^{a}$ Values are presented as the mean \pm standard error of the mean of two independent experiments. $\mu_{\max }$, maximum specific growth rate $\left(\times 10^{-2} \mathrm{~h}^{-1}\right) ; K_{\mathrm{S}}$, half saturation constant, $\left(\mathrm{mg}_{\mathrm{P}} \mathrm{L}^{-1}\right)$; RMSE, root mean squared error $\left(\times 10^{-2} \mathrm{~h}^{-1}\right)$; $\% \mathrm{SEP}$, standard error of prediction; $B_{\mathrm{f}}$, bias factor; $A_{\mathrm{f}}$, accuracy factor. 
to the production of allelochemicals. In fact, lower biomass productivities determined for this microalga in co-cultures suggest that unlike mono-cultures, nutrients removal was devoted to the production of other molecules, rather than microalgal biomass. The excretion of metabolic molecules and harmful chemicals presenting inhibitory effects towards cyanobacteria or microalgae in co-cultures has already been reported in the literature..$^{\mathbf{2 0 , 2 1 , 5 0 , 5 1}}$ Moreover, BittencourtOliveira $e t$ al. ${ }^{50}$ suggested that nutrient limitation is not the only factor that can explain the prevalence of a given strain in co-cultures. The presence of allelochemicals can also regulate the interaction of these microorganisms. ${ }^{50}$

Fig. 2A and $\mathrm{C}$ show the modified Gompertz model fits obtained for mono-cultures of $S$. salina and $P$. subcapitata, respectively. Differences in initial biomass concentrations between both microorganisms were related to the different cell densities of the microorganisms, as all the cultures were inoculated with the same initial cellular concentration (between 1 and $2 \times 10^{6}$ cells per $\mathrm{mL}$ ). The closeness of the fits obtained through the modified Gompertz model can be evaluated by observing the model curves superimposed on the experimental data, which means that the modified Gompertz model correctly describes the behaviour of the selected microorganisms. In fact, low RMSE and \% SEP values were determined for all the model fits (Table 3). In addition, the values of $B_{\mathrm{f}}$ and $A_{\mathrm{f}}$ close to one (Table 3 ) also confirm the existence of a good correlation between estimated values and experimental data.

Biological parameters, such as lag time, $\lambda$, and upper asymptote value, $A$, determined for $S$. salina and $P$. subcapitata grown in mono-cultures are shown in Table 3. Values of lag time determined for these microorganisms were negative, indicating that both cultures were acclimated to the experimental conditions. These results were not surprising since both $S$. salina and $P$. subcapitata were acclimated to phosphorus concentrations
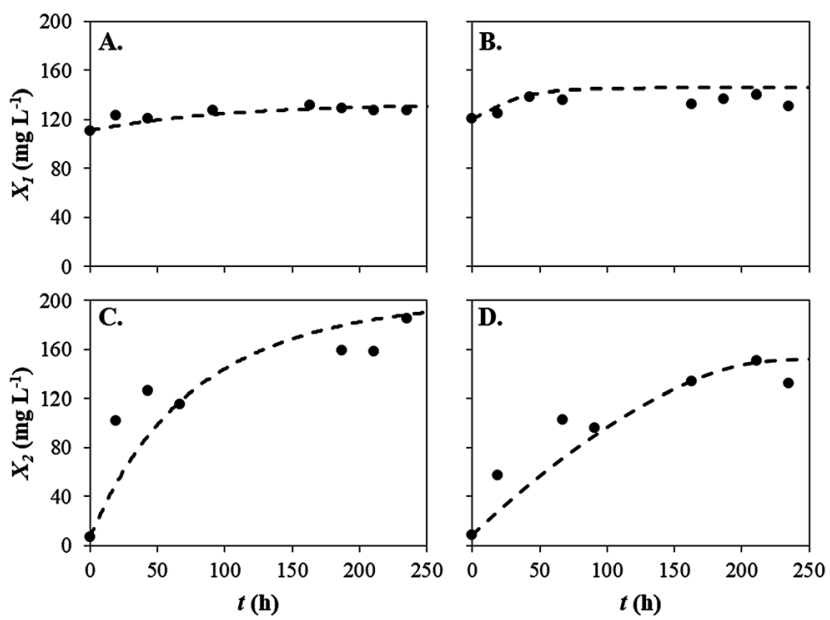

Fig. 2 Model fit of the modified Gompertz model to the experimental data: (A) S. salina grown in mono-cultures; (B) S. salina grown in cocultures; (C) P. subcapitata grown in mono-cultures; (D) P. subcapitata grown in co-cultures. Dashed lines represent the predicted values obtained through the modified Gompertz model.

Table 3 Kinetic parameters and performance indexes of the modified Gompertz model for mono- and co-cultures of $S$. salina and P. subcapitata ${ }^{a}$

Mono-cultures Co-cultures

S. salina $\quad$ P. subcapitata $\quad$ S. salina $\quad$ P. subcapitata

\begin{tabular}{lllll}
\hline$\lambda(\mathrm{h})$ & $<0$ & $<0$ & - & - \\
$A\left(\mathrm{mg} \mathrm{L}^{-1}\right)$ & 400 & 418 & - & - \\
$\gamma$ & - & - & 33.0 & \\
$\beta$ & - & - & 0.710 & \\
$\operatorname{RMSE}\left(\mathrm{mg} \mathrm{L}^{-1}\right)$ & 4 & 30 & 9 & 17 \\
$\% \mathrm{SEP}$ & 3 & 22 & 7 & 17 \\
$B_{\mathrm{f}}$ & 0.990 & 0.886 & 1.06 & 0.880 \\
$A_{\mathrm{f}}$ & 1.02 & 1.22 & 1.06 & 0.18
\end{tabular}

${ }^{a} \lambda$, lag time (h); $A$, maximum biomass concentration or upper asymptote value $\left(\mathrm{mg} \mathrm{L}^{-1}\right) ; \gamma$, measure of the inhibitory effect of the allelochemicals produced by $P$. subcapitata; $\beta$, fraction of potential growth devoted to the production of allelochemicals; RMSE, root mean squared error $\left(\mathrm{mg} \mathrm{L}^{-1}\right)$; \% SEP, standard error of prediction; $B_{\mathrm{f}}$, bias factor; $A_{\mathrm{f}}$, accuracy factor.

within the range used in this study prior to the mono- and coculture experiments. Additionally, low $\lambda$ values, approximately 4-5 $\mathrm{h}$, or even negative values were obtained in the studies performed by Çelekli et $a .^{39}$ Regarding maximum biomass concentrations, $A$, the values determined for $S$. salina and $P$. subcapitata were 400 and $418 \mathrm{mg} \mathrm{L}^{-1}$, respectively. These maximum values indicate the biomass concentration achieved when stationary growth phase was reached. Both microorganisms reached the stationary growth phase after $67 \mathrm{~h}$ of culturing.

Fixing $\lambda$ and $A$ values determined for mono-cultures, the parameters $\beta$ and $\gamma$ were determined according to eqn (9). Fig. 2B and D show the growth curves obtained for $S$. salina and $P$. subcapitata in co-cultures and the respective model fits. The positive parameter value obtained for the measure of the inhibitory effect of the allelochemicals produced by $P$. subcapitata, $\gamma$, confirms the hypothesis of growth inhibition of $S$. salina by allelochemicals released by the microalga (Table $3)$. Although the production of allelochemicals by this microalga is not documented in the literature, it has already been reported for other freshwater species, such as C. vulgaris, ${ }^{\mathbf{2}}$ Botryococcus braunii, ${ }^{53}$ S. obliquus ${ }^{54}$ and Chlamydomonas reinhardtii. ${ }^{55}$ In the study performed by Fergola et al.,${ }^{21} \gamma$ value estimated for the assessment of the inhibitory effect of C. vulgaris towards P. subcapitata was 7.81. The fraction of potential growth devoted to allelochemicals production, represented by $\beta$, was estimated to be 0.710 , which indicates that a large amount of phosphate-phosphorus present in the culture medium is used by $P$. subcapitata to produce allelochemicals. These results corroborate the low average biomass productivities determined for $P$. subcapitata grown in co-cultures. According to Fergola et al. ${ }^{21}$ if $0<\beta<1$, the competition is driven towards the extinction of the strain that presents lower biomass productivities. In this study, biomass productivities determined for $S$. salina grown in co-cultures were lower than those determined for $P$. subcapitata, 


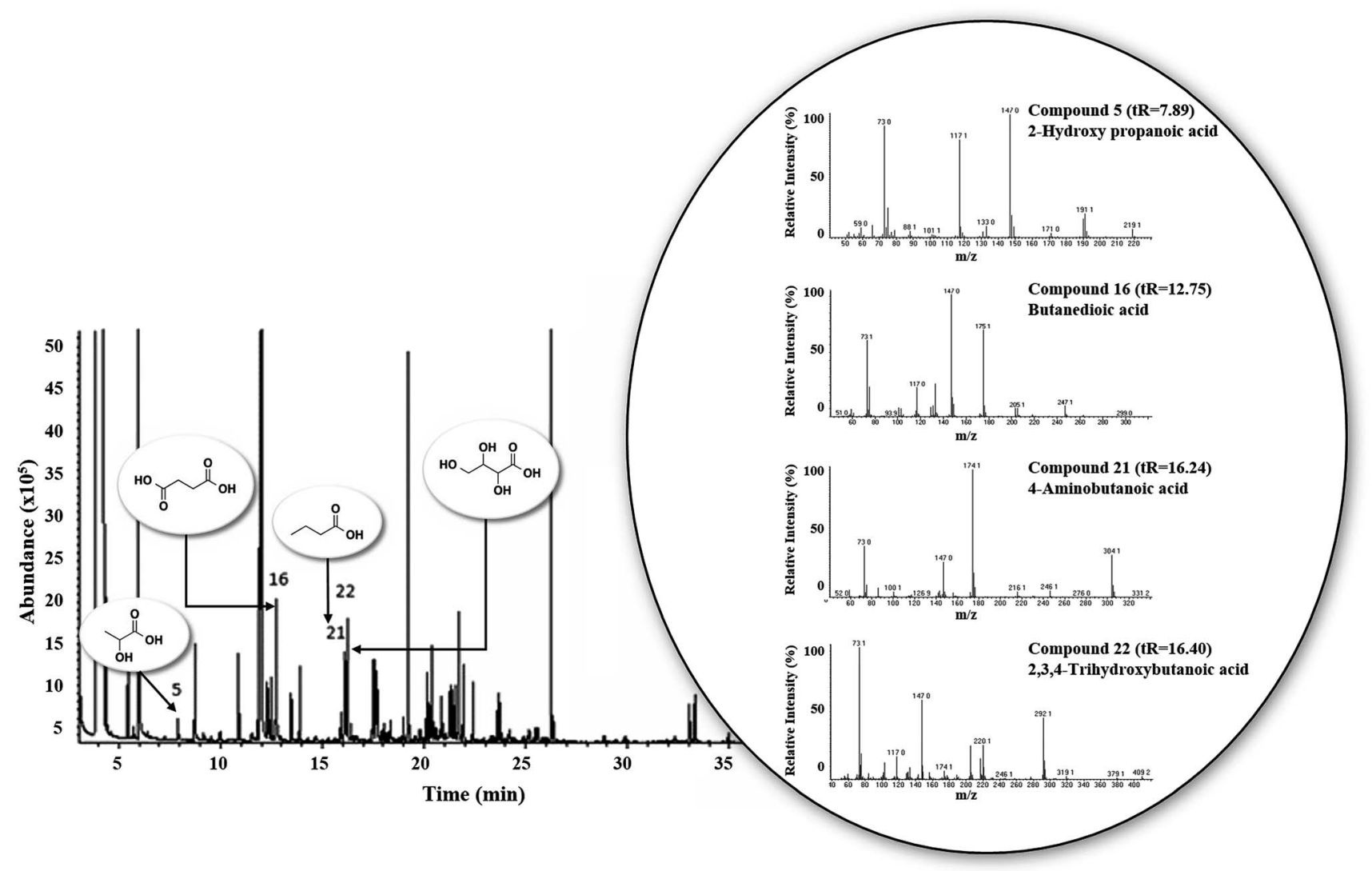

Fig. 3 GC-MS chromatogram of the co-cultures medium of S. salina and P. subcapitata. Peaks 5, 16, 21 and 22 correspond to 2-hydroxypropanoic acid, butanedioic acid, 4-aminobutanoic acid and 2,3,4-trihydroxybutanoic acid, respectively. The mass spectra correspond to the organic acids silane derivatives.

meaning that its growth inhibition was promoted by allelochemicals produced by the co-cultivated microalga.

\subsection{Co-cultures medium analysis and evaluation of the inhibitory activity of identified allelochemicals}

The analysis of the supernatant of $S$. salina with $P$. subcapitata co-cultures by GC-MS and 1D-NMR demonstrated the presence of several metabolites, such as alkaloids, amino acids, organic acids, sugars (mono- and disaccharides) and alcohols (see ESI, File $\mathrm{S} 4 \dagger$ ). Excretion of this type of compounds in microalgae and cyanobacteria polycultures has already been described. ${ }^{\mathbf{5 6 - 6 2}}$

Four organic acids (2-hydroxypropanoic acid - 5, butanedioic acid - 16, 4-aminobutanoic acid - 21 and 2,3,4-trihydroxybutanoic acid - 22), identified from GC-MS analysis (Fig. 3), were selected for an in-depth growth inhibitory study. In fact, several studies have pointed out that this type of organic acids can act as effective antimicrobial agents; ${ }^{\mathbf{6 3 - 6 7}}$ therefore it was decided to inspect their effects on the growth of each microorganism. Accordingly, their inhibitory potential towards $S$. salina and $P$. subcapitata was evaluated (see ESI, File S5 $\dagger$ ). Results have shown that all the organic acids tested had no inhibitory effect on the growth of $P$. subcapitata and $S$. salina, except 2-hydroxypropanoic acid (5). Lactic acid (2-hydroxypropanoic acid (5)) displayed an inhibitory growth activity on $S$. salina, but not on
P. subcapitata, suggesting the role of this organic acid as an allelochemical able to modify the growth of $S$. salina. This result corroborates the data obtained with the modified Gompertz model, by which it was proposed that the inhibition of $S$. salina growth was a consequence of the presence of allelochemicals excreted by $P$. subcapitata.

\section{Conclusions}

The behaviour of $S$. salina and $P$. subcapitata under low phosphate-phosphorus concentrations was assessed by studying their growth in mono- and co-cultures. For increasing phosphorus concentrations, higher average biomass productivities were determined for both microorganisms. However, lower values were determined in co-cultures. Regarding specific growth rates, values determined for both microorganisms were higher for increased phosphorus concentrations, being constant for higher nutrient concentrations. This behaviour was correctly described by the Monod model fitted to the experimental data. Higher specific growth rates were obtained for the microalga (both in mono- and co-cultures), indicating that this microorganism presents higher ability to uptake phosphorus supplied at low levels. Regarding $S$. salina, the specific growth rates determined in co-cultures were significantly lower than those obtained in mono-cultures. Data 
coming from the development of the modified Gompertz model suggested that growth inhibition of $S$. salina in co-cultures was related to the presence of allelochemicals produced by $P$. subcapitata. Metabolomic and antimicrobial analysis demonstrated that lactic acid (2-hydroxypropanoic acid) can be proposed as an allelochemical involved in growth inhibition of $S$. salina when co-cultured with $P$. subcapitata. This study provides new insights on allelochemical production by the freshwater microalga $P$. subcapitata and how they can influence the growth of other species, such as $S$. salina. This information can be very useful to maintain naturally-occurring species in natural lakes or ponds and in aquaculture. Additionally, this study proposes simple methods for the understanding of interactions involved in co-cultures.

\section{Acknowledgements}

This work was financially supported by: Project POCI-01-0145FEDER-006939 - Laboratory for Process Engineering, Environment, Biotechnology and Energy - LEPABE funded by FEDER funds through COMPETE2020 - Programa Operacional Competitividade e Internacionalização (POCI) - and by national funds through FCT - Fundação para a Ciência e a Tecnologia; Project 0687-Novomar-1-P; Scholarships SFRH/BD/88799/2012, SFRH/BD/84383/2012 and SFRH/BPD/66721/2009. Dr A. Coqueiro thanks Ciências sem fronteiras, CAPES Foundation, Ministry of Education of Brazil, for the scholarship. The authors also acknowledge CIIMAR (Centre of Marine and Environmental Research of the University of Porto), for providing the cyanobacterium Synechocystis salina LEGE 06079.

\section{References}

1 L. Brennan and P. Owende, Renewable Sustainable Energy Rev., 2010, 14, 557-577.

2 A. Demirbas, Appl. Energy, 2011, 88, 3541-3547.

3 D. Bilanovic, A. Andargatchew, T. Kroeger and G. Shelef, Energy Convers. Manage., 2009, 50, 262-267.

4 S.-H. Ho, C.-Y. Chen, D.-J. Lee and J.-S. Chang, Biotechnol. Adv., 2011, 29, 189-198.

5 R. Sayre, Bioscience, 2010, 60, 722-727.

6 J. C. M. Pires, A. L. Gonçalves, F. G. Martins, M. C. M. AlvimFerraz and M. Simões, Mitigation and Adaptation Strategies for Global Change, 2013, 19, 1109-1117.

7 N. C. Boelee, H. Temmink, M. Janssen, C. J. N. Buisman and R. H. Wijffels, Water Res., 2011, 45, 5925-5933.

8 J. B. K. Park, R. J. Craggs and A. N. Shilton, Bioresour. Technol., 2011, 102, 35-42.

9 I. Rawat, R. Ranjith Kumar, T. Mutanda and F. Bux, Appl. Energy, 2011, 88, 3411-3424.

10 A. Silva-Benavides and G. Torzillo, J. Appl. Phycol., 2012, 24, 267-276.

11 Q. Hu, in Handbook of Microalgal Culture: Biotechnology and Applied Phycology, ed. A. Richmond, Blackwell Science Ltd, Oxford, UK, 2004, ch. 12, pp. 268-271.

12 S. Singh, B. Kate and U. Banerjee, Crit. Rev. Biotechnol., 2005, 25, 73-95.
13 P. Spolaore, C. Joannis-Cassan, E. Duran and A. Isambert, J. Biosci. Bioeng., 2006, 101, 87-96.

14 A. D. Cembella, Phycologia, 2003, 42, 420-447.

15 F. D. Hulot, P. J. Morin and M. Loreau, Oikos, 2001, 95, 231238.

16 S. R. Subashchandrabose, B. Ramakrishnan, M. Megharaj, K. Venkateswarlu and R. Naidu, Biotechnol. Adv., 2011, 29, 896-907.

17 S. Boonma, S. Chaiklangmuang, S. Chaiwongsar, J. Pekkoh, C. Pumas, T. Ungsethaphand, S. Tongsiri and Y. Peerapornpisal, Clean: Soil, Air, Water, 2014, 43, 761-766. 18 E. Fouilland, Rev. Environ. Sci. Bio/Technol., 2012, 11, 1-4.

19 K. R. Johnson and W. Admassu, J. Chem. Technol. Biotechnol., 2013, 88, 992-998.

20 E. M. Gross, Crit. Rev. Plant Sci., 2003, 22, 313-339.

21 P. Fergola, M. Cerasuolo, A. Pollio, G. Pinto and M. DellaGreca, Ecol. Modell., 2007, 208, 205-214.

22 A. Çelekli, M. Yavuzatmaca and H. Bozkurt, Bioresour. Technol., 2009, 100, 3625-3629.

23 F. Mairet, O. Bernard, T. Lacour and A. Sciandra, Proceedings of the 18th World Congress of The International Federation of Automatic Control, Milano, Italy, 28 August-2 September 2011.

24 C. Zonneveld, Ecol. Modell., 1998, 113, 41-54.

25 L. Barsanti and P. Gualtieri, Algae - Anatomy, Biochemistry and Biotechnology, CRC Press, USA, 2nd edn, 2006.

26 A. Kumar, S. Ergas, X. Yuan, A. Sahu, Q. Zhang, J. Dewulf, F. X. Malcata and H. Van Langenhove, Trends Biotechnol., 2010, 28, 371-380.

$27 \mathrm{Q}$. Hu, P. Westerhoff and W. Vermaas, Appl. Environ. Microbiol., 2000, 66, 133-139.

28 OECD, Test Guideline 201, Organisation for economic cooperation and development, 2011.

29 A. Gonçalves, M. Simões and J. Pires, Energy Convers. Manage., 2014, 85, 530-536.

30 B. Kolar, L. Arnuš, B. Jeretin, A. Gutmaher, D. Drobne and M. K. Durjava, Chemosphere, 2014, 115, 75-80.

31 I. Rodea-Palomares, K. Boltes, F. Fernández-Piñas, F. Leganés, E. García-Calvo, J. Santiago and R. Rosal, Toxicol. Sci., 2011, 119, 135-145.

32 P. Feng, Z. Deng, L. Fan and Z. Hu, J. Biosci. Bioeng., 2012, 114, 405-410.

33 E. Jacob-Lopes, C. H. G. Scoparo, L. M. C. F. Lacerda and T. T. Franco, Chem. Eng. Process., 2009, 48, 306-310.

34 J. Monod, Annu. Rev. Microbiol., 1949, 3, 371-394.

35 D. M. Di Toro, Ecol. Modell., 1980, 8, 201-218.

36 R. W. Sterner and J. P. Grover, Water Res., 1998, 32, 35393548.

37 L. Xin, H. Hong-ying, G. Ke and S. Ying-xue, Bioresour. Technol., 2010, 101, 5494-5500.

38 B. Gompertz, Philos. Trans. R. Soc. London, 1825, 115, 513-583. 39 A. Çelekli, M. Balcı and H. Bozkurt, Bioresour. Technol., 2008, 99, 8742-8747.

40 L. M. C. F. Lacerda, M. I. Queiroz, L. T. Furlan, M. J. Lauro, K. Modenesi, E. Jacob-Lopes and T. T. Franco, J. Pet. Sci. Eng., 2011, 78, 679-686.

41 Y. Chisti, Biotechnol. Adv., 2007, 25, 294-306. 
42 S. C. Chapra and R. P. Canale, Numerical Methods for Engineers, McGraw-Hill Higher Education, New York, 6th edn, 2010.

43 M. I. Queiroz, M. O. Hornes, A. G. da Silva-Manetti and E. Jacob-Lopes, Appl. Energy, 2011, 88, 3438-3443.

44 F.-M. Li and H.-Y. Hu, Appl. Environ. Microbiol., 2005, 71, 6545-6553.

45 L. Ni, K. Acharya, X. Hao and S. Li, Chemosphere, 2012, 88, 1051-1057.

46 A. W. Bauer, M. D. K. Kirby, J. C. Sherria and M. Turck, Am. J. Clin. Pathol., 1966, 45, 493-506.

47 E. Litchman, D. Steiner and P. Bossard, Freshwater Biol., 2003, 48, 2141-2148.

48 F.-X. Fu, Y. Zhang, P. R. F. Bell and D. A. Hutchins, J. Phycol., 2005, 41, 62-73.

49 M. Zwietering, I. Jongenburger, F. Rombouts and K. Van't Riet, Appl. Environ. Microbiol., 1990, 56, 1875-1881.

50 M. C. Bittencourt-Oliveira, M. A. Chia, H. S. B. Oliveira, M. K. C. Araújo, R. J. R. Molica and C. T. S. Dias, J. Appl. Phycol., 2014, 27, 275-284.

51 J. Leflaive and L. Ten-Hage, Freshwater Biol., 2007, 52, 199214.

52 R. Pratt and J. Fong, Am. J. Bot., 1940, 27, 431-436.

53 I.-Z. Chiang, W.-Y. Huang and J.-T. Wu, J. Phycol., 2004, 40, 474-480.
54 X.-H. Jia, D.-J. Shi, R.-J. Kang, H.-M. Li, Y. Liu, Z.-Z. An, S.-S. Wang, D.-H. Song and G.-S. Du, in Photosynthesis. Energy from the Sun, Springer, 2008, pp. 1339-1342.

55 V. W. Proctor, Limnol. Oceanogr., 1957, 2, 123-139.

56 R. A. Lewin, Can. J. Microbiol., 1956, 2, 665-672.

57 M. B. Allen, Arch. Mikrobiol., 1956, 24, 163-168.

58 A. Mishra, K. Kavita and B. Jha, Carbohydr. Polym., 2011, 83, 852-857.

59 J. A. Hellebust, Limnol. Oceanogr., 1965, 10, 192-206.

60 H. Fallowfield and M. Daft, British Phycological Journal, 1988, 23, 317-326.

61 E. Granum, S. Kirkvold and S. M. Myklestad, Mar. Ecol.: Prog. Ser., 2002, 242, 83-94.

62 A. M. Waite, R. J. Olson, H. G. Dam and U. Passow, J. Phycol., 1995, 31, 925-933.

63 Y.-W. In, J.-J. Kim, H.-J. Kim and S.-W. Oh, J. Food Saf., 2013, 33, 79-85.

64 S. A. Ibrahim, H. Yang and C. W. Seo, Food Chem., 2008, 109, 137-143.

65 J. L. Thompson and M. Hinton, Br. Poult. Sci., 1997, 38, 5965.

66 S. Doores, in Antimicrobials in Food, ed. P. M. Davidson, J. N. Sofos and A. L. Branen, CRC Press, Florida, USA, 2005, ch. 4, pp. 91-142.

67 C. B. Huang, Y. Alimova, T. M. Myers and J. L. Ebersole, Arch. Oral Biol., 2011, 56, 650-654. 